\title{
Medición y Certificación de Niveles de Intensidad de Campos Electromagnéticos No Ionizantes en Ambientes Clínico Hospitalarios
}

\author{
Measurement and Certification of Intensity Levels of Non-Ionizing \\ Electromagnetic Fields in Clinical and Hospital Environments. \\ Luis Enrique Llamosa Rincón ${ }^{1}$, Vanessa Díaz Izquierdo², Daniela Cardona Clavijo² \\ ${ }^{1}$ Departamento de Matemáticas, Grupo de Investigación de Electrofisiología Universidad Tecnológica de \\ Pereira, Pereira, Colombia. \\ Correo-e: lellamo@utp.edu.co \\ ${ }^{2}$ Ingeniería Física, Joven Investigador Colciencias, Grupo de Investigación de Electrofisiología Universidad \\ Tecnológica de Pereira, Pereira, Colombia. \\ Correo-e: etruizorduis.edu.co
}

\begin{abstract}
Resumen- El incremento de la telefonía celular y el aumento de sistemas inalámbricos de diagnóstico y transmisión de datos dentro del ámbito hospitalario, originan contaminación de campos electromagnéticos (CEM) en áreas críticas de los hospitales; estos CEMs pueden interferir en el correcto funcionamiento de los equipos electromédicos; en este artículo se analizan los aspectos fundamentales para el diseño e implementación de protocolos de medición y certificación de niveles de intensidad de campos electromagnéticos no ionizantes de alta y baja frecuencia, aplicables a ambientes clínicohospitalarios, acordes con la normatividad nacional e internacional existente para tal fin; como resultado más importante se obtuvo el diseño e implementación de los protocolos de medición, teniendo en cuenta que estos cumplen con los requisitos de competencia técnica necesarios para realizar las respectivas mediciones.
\end{abstract}

Palabras clave- Ambientes hospitalarios, medición y certificación, ondas electromagnéticas, protocolos técnicos.

Abstract - The growth of wireless communications, radio and worldwide basis in clinical and hospital environments stations, create an exposure to non-ionizing electromagnetic radiation of high and low frequencies, that can create interference with medical equipment, which can be detrimental to these. This article discusses the fundamental aspects for the design and implementation of measurement and certification protocols of levels of intensity non-ionizing electromagnetic fields (FEM) of high and low frequency, applicable to clinical hospitable environments consistent with existing national and international standards were studied to this purpose. The most important result was the design and implementation of measurement protocols, considering that they comply with the requirements of technical competence required to perform such measurements.

Key Word - Electromagnetic waves, hospital environments, measurement and certification, technical protocols.

\section{INTRODUCCIÓN}

El uso de diversas tecnologías que generan campos electromagnéticos artificiales, en ambientes hospitalarios, ha sido tema de interés a nivel mundial ya que estas interferencias electromagnéticas pueden afectar el correcto funcionamiento de los equipos electromédicos, arrojando un diagnostico poco confiable para el paciente. La compatibilidad electromagnética se refiere a la capacidad que tiene un equipo para trabajar adecuadamente en ambientes expuestos a campos electromagnéticos. La Compatibilidad electromagnética de los dispositivos y equipos eléctricos, electrónicos y de telecomunicaciones, es hoy en día a escala mundial, una de las principales exigencias de calidad en ambientes clínico-hospitalarios.

Para el estudio de la compatibilidad electromagnética hay que analizar tres aspectos: el emisor o fuente de la interferencia, el 
mecanismos de acoplamiento y el receptor o víctima de la interferencia como lo explica la Figura 1.

La interferencia electromagnética (EMI) es la presencia de voltajes parásitos o corrientes no deseadas que pueden aparecer en un equipo o en sus circuitos; entre los emisores que se pueden encontrar en un ambiente hospitalario tenemos: equipos de telecomunicaciones (teléfonos móviles, internet inalámbrico, etc.), escáner de resonancia magnética, equipos de monitoreo a distancia entre otros. El camino de acoplamiento está divido en acoplamiento radiado producto de la radiación electromagnética por una transferencia de energía entre dos o más circuitos y acoplamiento conducido dado por la propagación entre conductores eléctricos. Entre los receptores se encuentra cualquier equipo electromédico sensible: monitores multi-parámetros, bombas de infusión, electro-ventiladores, marcapasos, computadoras, entre otros.

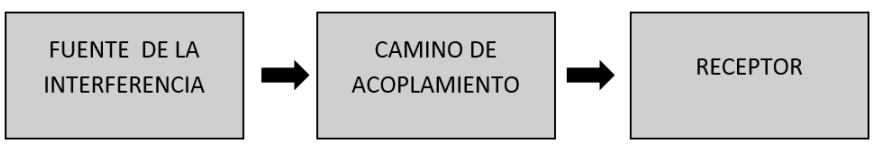

Figura 1. Bloques funcionales de interferencia.

Las interferencias electromagnéticas (EMI), pueden ser un problema considerable para cualquier dispositivo electrónico, pero en los dispositivos médicos, las consecuencias pueden ser fatales. En el Reino Unido, el "Medical Device Agency y en Canadá el Health Canada's Medical Devices Bureau" ha realizado registros de este tipo de incidentes. Los problemas con este origen registrados por la "Food and Drug Administration" (FDA) de los EEUU, desde 1979 incluyen fallos debido a EMI conducidas y radiadas, alteraciones producidas por líneas de alta tensión y por descargas electrostáticas. Todos estos casos destacan la necesidad de incrementar las precauciones adoptadas por parte de usuarios, ingenieros, fabricantes, investigadores y organismos reguladores. Así mismo el "Health Canada's Medical Devices Bureau" recibió entre los años 1984 y 2000, treinta y seis informes de fallos de funcionamiento de productos sanitarios atribuidos a interferencias electromagnéticas.

La norma IEC 60601-1-2, establece que cada equipo electromédico debe poseer un documento acompañante, el cual es un informe en donde se especifica por parte del fabricante, las precauciones y advertencias con respecto a compatibilidad electromagnética que posee cada equipo.

Es requisito de ley en muchos organismos regulatorios nacionales que para la homologación de los equipos electromédicos, cada fabricante o importador presente su documento acompañante ante los entes competentes, así cada usuario toma las precauciones esenciales para la seguridad y fiabilidad de los dispositivos médicos electrónicos. En la tabla
1 se presentan algunos ejemplos de incidencias médicas registradas por dispositivos médicos y su fuente de origen [1].

En este artículo se presenta el diseño e implementación de protocolos para la medición y certificación de niveles de intensidad de campos electromagnéticos no ionizantes en ambientes clínico-hospitalarios; estas mediciones se llevaron a cabo con el fin de verificar si el hospital en estudio cumplía con los niveles fijados con el estándar IEC 60601-1-2 (IEC 2004), el cual establece que los equipos electromédicos no se pueden someter a un campo magnético superior a $3 \mathrm{~A} / \mathrm{m}$, para campo eléctrico este estándar establece que los equipos de monitoreo crítico y asistencia vital deben soportar una interferencia radiada de hasta $10 \mathrm{~V} / \mathrm{m}$ y en el caso de los equipos de monitoreo no crítico, el nivel que deben soportar es de $3 \mathrm{~V} / \mathrm{m}[2]$.

\begin{tabular}{|l|l|}
\hline DISPOSITIVO MÉDICO & FUENTE DE ORIGEN \\
\hline Monitores de apnea & Radiodifusión en FM \\
\hline $\begin{array}{l}\text { Monitores de gas de } \\
\text { anestesia }\end{array}$ & Electrobisturíes \\
\hline Electrocardiograma & $\begin{array}{l}\text { Telefonía móvil celular } \\
\text { analógica y digital }\end{array}$ \\
\hline $\begin{array}{l}\text { Bombas de infusión y y } \\
\text { jeringa }\end{array}$ & $\begin{array}{l}\text { Telefonía móvil celular } \\
\text { analógica y digital y } \\
\text { equipos de rayos X } \\
\text { portátiles }\end{array}$ \\
\hline Sillas de ruedas eléctricas & $\begin{array}{l}\text { Equipos de comunicaciones } \\
\text { de policía, bomberos y } \\
\text { radioaficionados }\end{array}$ \\
\hline Análisis hematológicos & Busca personas \\
\hline $\begin{array}{l}\text { Indicación de temperatura y } \\
\text { presión sanguínea }\end{array}$ & Electrobisturíes \\
\hline Monitores de incubadoras & $\begin{array}{l}\text { Radioaficionados, telefonía } \\
\text { móvil celular }\end{array}$ \\
\hline Marcapasos & $\begin{array}{l}\text { Comunicaciones de } \\
\text { ambulancia, walky-talkies, } \\
\text { detector de metales }\end{array}$ \\
\hline $\begin{array}{l}\text { Monitor de telemetría } \\
\text { cardiaco }\end{array}$ & $\begin{array}{l}\text { Comunicaciones en 160- } \\
174 \text { MHz }\end{array}$ \\
\hline Respirador & $\begin{array}{l}\text { Equipos de rayos X } \\
\text { portátiles, walky- talkies y } \\
\text { radiodifusión en FM }\end{array}$ \\
\hline Telefonía móvil celular \\
\hline Equipos de diálisis & Telefonía móvil celular \\
\hline Desfibriladores & $\begin{array}{l}\text { Pagging } \\
\text { máky-talkies, telefonía }\end{array}$ \\
\hline Monitor de telemetría & Electrobisturíes \\
\hline Ayudas a la audición & \\
\hline Equipos de laparoscopia & \\
\hline
\end{tabular}

Tabla 1. Incidencias Médicas registradas por dispositivos médicos y su fuente de origen. 


\section{MATERIALES Y MÉTODOS}

Los centros de salud deben tener normativas que permitan controlar, monitorear y evaluar sus equipos médicos y su entorno electromagnético a fin de prevenir posibles errores en diagnóstico y minimizar riesgos de operatividad, es por ello que se adoptan estándares establecidos, como: NTC-ISO/IEC 17025 "requisitos generales para la competencia de los laboratorios de ensayo y calibración"

[4], GTC 51 "guía para la expresión de incertidumbres en las mediciones" [5], Norma Técnica Colombiana NTC- IEC 60601-1-2 "Requisitos generales para la seguridad básica y el funcionamiento esencial de equipos electromédicos" [6], ICNIRP (International Comission on Non-Ionizing Radiation Protection) [7].

Este estudio se realizó en el hospital San Vicente de Paúl en Santa Rosa de Cabal, Risaralda, Colombia, en cuatro salas que el hospital puso a disposición para esta investigación, las cuales fueron: sala de procedimientos, sala de reanimación, sala de maternidad y sala de parto.

En las mediciones fueron usados dos equipos de banda ancha, el primero es el medidor de campo magnético ELT 400 de baja frecuencia el cual tiene una resolución de medida de $0.001 \%$, un rango de frecuencia de $1 \mathrm{~Hz} \mathrm{a} 400 \mathrm{kHz}$ y posee una sonda isotrópica de $100 \mathrm{~cm} 2$ [8], el segundo instrumento de medida es el NBM 520 para alta frecuencia, el cual tiene una resolución de medida de $2 \%$, un rango de frecuencia de $100 \mathrm{kHz}$ a $60 \mathrm{GHz}$ y cuenta con dos sondas isotrópicas, una para medir campo eléctrico y otra para medir campo magnético [9].

La mejor técnica para realizar la medición de campo electromagnético en lugares amplios que poseen una variedad de fuentes de radiación es la de mapeo electromagnético, esta técnica consiste en hacer un recorrido a lo largo y ancho del sitio con el objetivo de abarcar la mayor área posible, como lo muestra la Figura 2, con el fin de identificar los puntos con mayor intensidad de campo electromagnético, Los valores obtenidos son graficados en un mapa de contorno, como se observa en la Figura 9.

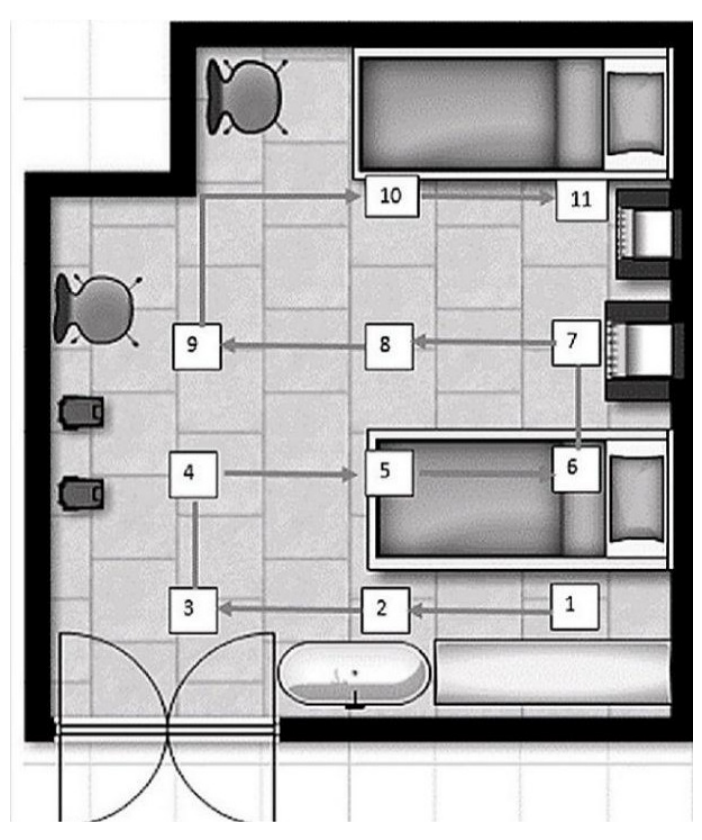

Figura 2. Modelo básico para el diagrama de mapeo electromagnético.

Las mediciones de campo eléctrico, campo magnético y densidad de potencia, fueron realizadas por el método de medición por inmisión, que consiste en la medición del campo electromagnético producto del aporte de múltiples fuentes que operan a distintas frecuencias. Para este tipo de medición se utilizan equipos de banda ancha. Esta medición tiene como objetivo obtener el nivel máximo de campo eléctrico, campo magnético o densidad de potencia, para lo cual se realiza un barrido de mediciones a velocidad lenta y constante [10].

Para llevar a cabo el protocolo de medición de campos electromagnéticos no ionizantes de alta frecuencia, se siguieron las recomendaciones de la norma UIT-TK. 52 de la unión internacional de telecomunicaciones [11], en donde se presenta una metodología que permite efectuar la evaluación y determinar la posibilidad de que una instalación o equipo sobrepase los límites de exposición a campos electromagnéticos no ionizantes, también se hace necesario tener en cuenta las recomendaciones establecidas por la ICNIRP [7].

Para realizar la implementación del protocolo de medida, se ubicó el instrumento de medida NBM520 en el punto escogido para iniciar el recorrido descrito en la Figura 2, las sondas de medida son conectadas al medidor, el cual se instala en un trípode no conductivo, para evitar errores de paralaje, se escogió el modo AVG para cada una de las componentes 
espectrales. Para cada punto elegido, se realizaron 4 medidas, cada una con intervalo de tiempo de 1 minuto, con el objetivo de analizar la variación en determinado punto. El trípode se ubicó a una distancia de 1,2 $\mathrm{m}$ con respecto a la superficie del suelo, esta altura se ha escogido como referente de investigaciones a nivel mundial [2], [10], realizando una medida secuencial entre punto y punto con una distancia de separación de $1 \mathrm{~m}$ entre los puntos de medición, abarcando la mayor área posible de cada habitación.

Para el procedimiento de medición de campos electromagnéticos no ionizantes de baja frecuencia, se lleva a cabo el procedimiento anteriormente mencionado, teniendo en cuenta que el instrumento de medida para este caso es el ELT 400, configurándolo en el modo RMS (valor eficaz), el cual surge de considerar corrientes sinusoidales; por recomendaciones estipuladas en el manual [8], las medidas deben hacerse con un ángulo de $45^{\circ}$ con respecto a la horizontal con el fin de recibir la mayor cantidad de radiación electromagnética posible.

Estimación de la incertidumbre de medición: Después de realizar las mediciones, se procede a establecer la metodología de estimación de la incertidumbre de medición de campos eléctricos, campos magnéticos y densidad de potencia, para alta y baja frecuencia, basada en la norma internacional GTC 51 "guía para la expresión de la incertidumbre en las mediciones" [5], la cual se basa en la GUM "guide to the expression of uncertainty in measurement" [12], a continuación se presenta la metodología utilizada para la estimación de la incertidumbre.

Incertidumbre tipo A: para una magnitud de entrada $X$ que varía aleatoriamente y que su valor se obtiene de $n$ observaciones repetidas, el mejor estimado $\bar{x}$ es la medida de las observaciones $x_{k}$ que se obtiene de la ecuación (1).

$$
x_{k}=\frac{1}{n} \sum_{k=1}^{n} x_{k}
$$

El mejor estimado de la incertidumbre estándar de tipo A $\left(U_{A}\right)$ es la desviación estándar $\left(S\left(x_{k}\right)\right)$ de la media de las observaciones (Ver ecuación 2).

$$
S\left(x_{k}\right)=\sqrt{\frac{1}{n-1} \sum_{k=1}^{n}\left(x_{k}-\bar{x}\right)^{2}}
$$

Por ende se obtiene la incertidumbre tipo A (ver ecuación 3):

$$
U_{A}=\frac{S\left(x_{k}\right)}{\sqrt{n}}
$$

Incertidumbre tipo $\boldsymbol{B}_{\mathbf{1}}$ : este tipo de incertidumbre no se obtiene por análisis estadístico de las mediciones, se obtiene por medio de información preexistente, para este caso se obtiene por especificaciones de exactitud del instrumento de medida de campos electromagnéticos (Ver ecuación 4).

$$
U_{B 1}=\frac{\text { Especificaciones de exactitud }}{\sqrt{3}}
$$

Incertidumbre tipo $\boldsymbol{B}_{2}$ : este tipo de incertidumbre no se obtiene por análisis estadístico de las mediciones, se obtiene por medio de información preexistente, para este caso la incertidumbre está asociada a la resolución de la indicación del instrumento de medición (Ver ecuación 5).

$$
U_{B 2}=\frac{\text { Resolución }}{2 \sqrt{3}}
$$

Incertidumbre estándar combinada $\boldsymbol{U}_{\boldsymbol{c}}$ : la incertidumbre combinada o total del resultado de una medición, se obtiene combinando apropiadamente las incertidumbres estándar de los estimados de las magnitudes de entrada. Esta incertidumbre se calcula como la raíz cuadrada positiva de la suma de los cuadrados de las incertidumbres tipo A y tipo B que intervienen en la medición. Para esto primero se debe encontrar el valor de los coeficientes de sensibilidad, pero como las medidas son directas y no están correlacionadas, los coeficientes de sensibilidad corresponden a 1 (Ver ecuación 6) [10].

$$
\mathrm{U}_{\mathrm{c}}=\sqrt{\left(\mathrm{U}_{\mathrm{A}}\right)^{2}+\left(\mathrm{U}_{\mathrm{B} 1}\right)^{2}+\left(\mathrm{U}_{\mathrm{B} 2}\right)^{2}}
$$

Después de obtener la incertidumbre combinada, se procede a elegir el nivel de confianza $\rho$, que para los protocolos diseñados fue establecido en $95 \%$; a continuación se evalúa el teorema del límite central [13]; para identificar si es posible la aplicación de este teorema se utiliza el criterio de la distribución dominante, llamando $U_{1}$ a la incertidumbre estándar de mayor magnitud y llamando $U_{R}$ a la combinación de las incertidumbres estándar restantes, como se muestra en las ecuaciones 7 y 8 .

$$
\begin{gathered}
U_{1}=U_{A}, U_{B 1}, U_{B 2} \quad \text { (la de mayor magnitud) } \\
U_{R}=U_{C}\left(\sin \text { incluir } U_{1}\right)
\end{gathered}
$$

El criterio de la distribución dominante se cumplió para las mediciones realizadas en la implementación de los protocolos diseñados, al satisfacer la ecuación 9, siendo para este caso la 
distribución dominante de manera general "rectangular", el factor de cobertura $K$ por lo tanto, tiene un valor de 1,65 [13].

$$
\frac{U_{R}}{U_{1}}<0.3
$$

Incertidumbre expandida $U_{E}$ : aunque la incertidumbre estándar combinada pueda utilizarse para expresar la incertidumbre del resultado de una medición en algunas aplicaciones, es necesario ofrecer una medida de la incertidumbre que represente a un intervalo alrededor del resultado de la medición dentro del cual puedan encontrarse los valores que razonablemente puedan ser atribuidos al mesurado con un alto nivel de confianza [10]. Para hallar la incertidumbre expandida se utilizó la ecuación 11.

$$
U_{E}=U_{c} * k
$$

Por último se expresa el resultado de la medida con su respectiva incertidumbre como se observa en la ecuación 12 .

$$
Y=y \pm U_{E}
$$

\section{RESULTADOS Y ANÁLISIS}

Teniendo en cuenta la normatividad internacional y nacional [4], [5], [6], [7], se realizó el diseño e implementación de los protocolos para la medición y certificación de campos electromagnéticos no ionizantes (CEM-NI) de alta y baja frecuencia en ambientes clínico-hospitalarios (como lo muestran las Figuras 3,4,5,6,7,8,9,10,11 para el caso de alta frecuencia), estos protocolos cumplen con los requisitos de competencia para realizar la medición de CEM-NI de alta y baja frecuencia, conforme a los estipulado en la norma NTC ISO IEC 17025 [4].
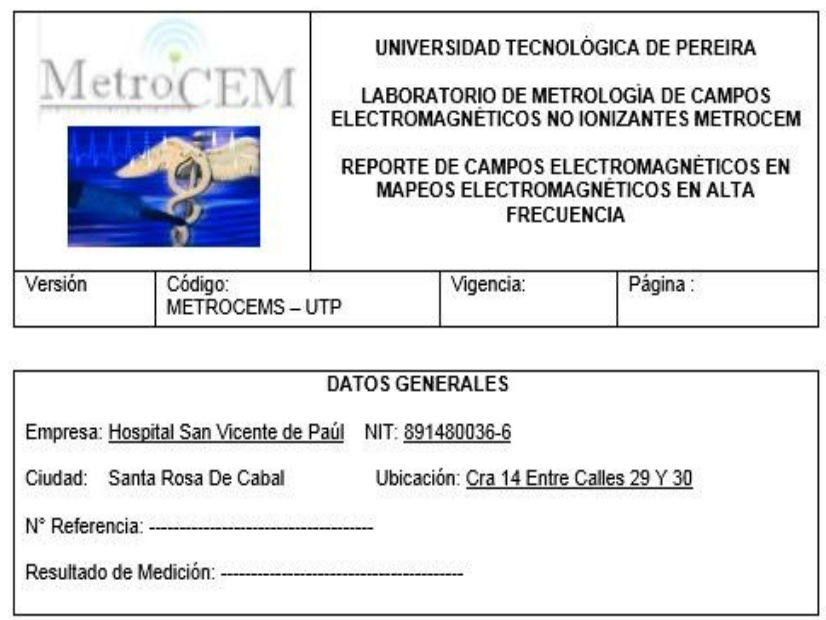

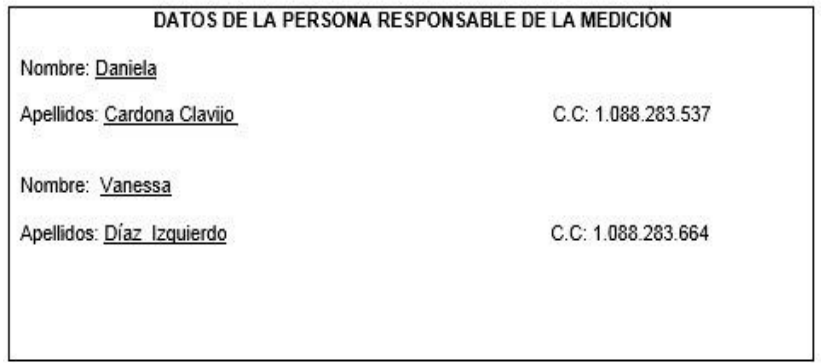

Figura 3. Formato de medida para la consignación de datos.

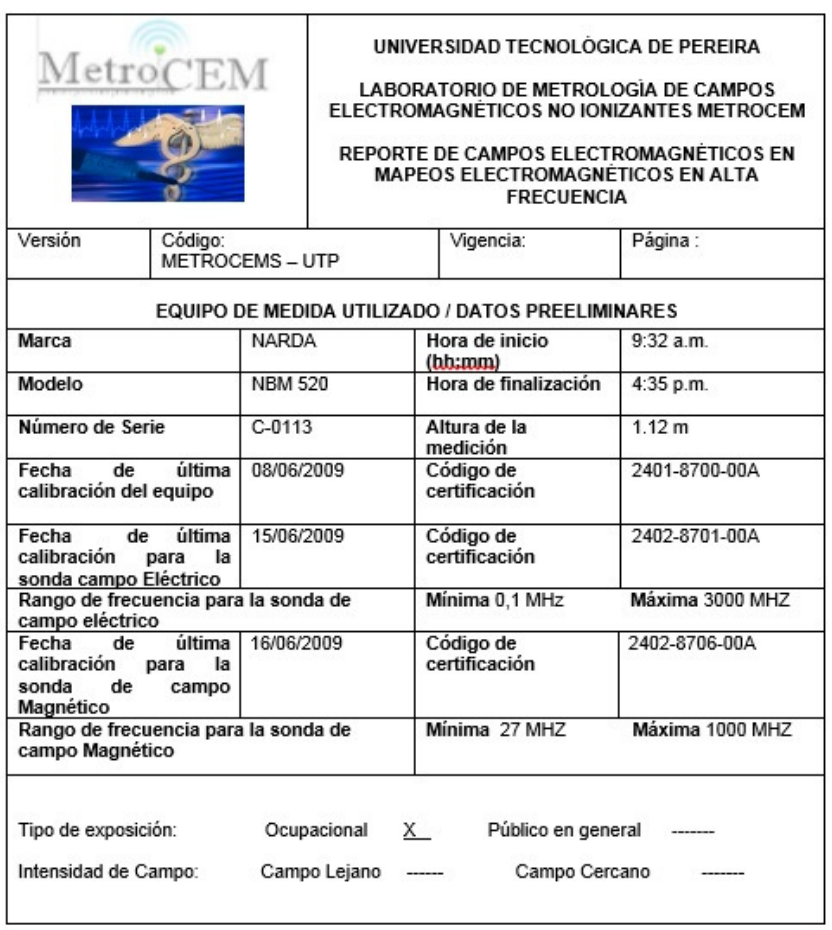

Figura 4. Formato de medida para la consignación de datos.

En la Figura 3 y la Figura 4, se presenta el formato de presentación del protocolo, en el cual se diligenciaron los 
datos generales del hospital San Vicente de Paúl en Santa Rosa de Cabal, Risaralda, Colombia, los datos de las personas encargadas de la medición y una breve descripción del instrumento utilizado para la medida. En la Figura 5, de manera fotográfica se muestra el diagrama de las instalaciones con sus fuentes de radiación, en la Figura 6 y Figura 7, se consignan los datos obtenidos para campo eléctrico, campo magnético y densidad de potencia de alta frecuencia.

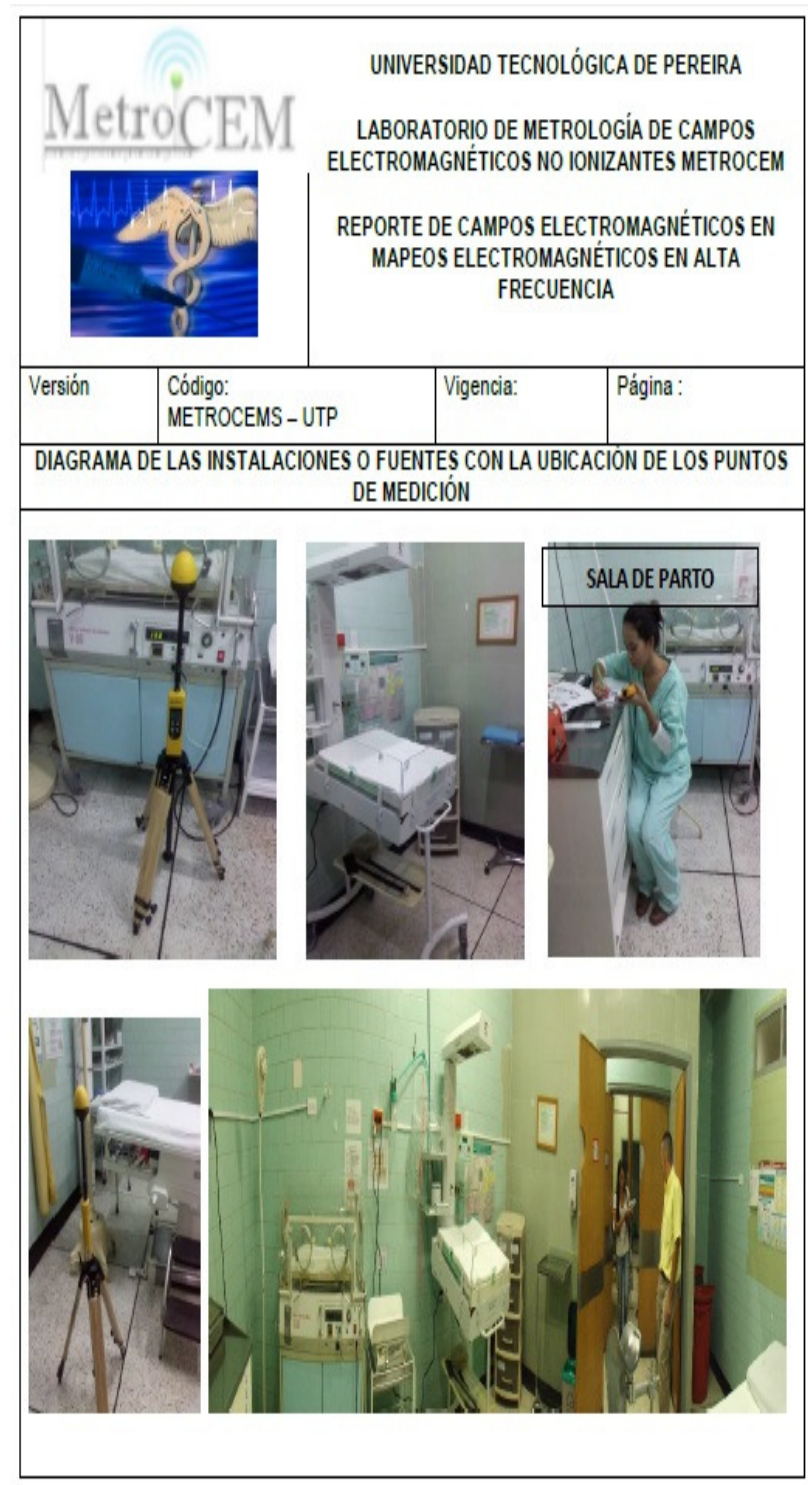

Figura 5. Formato de medida para la consignación de datos.

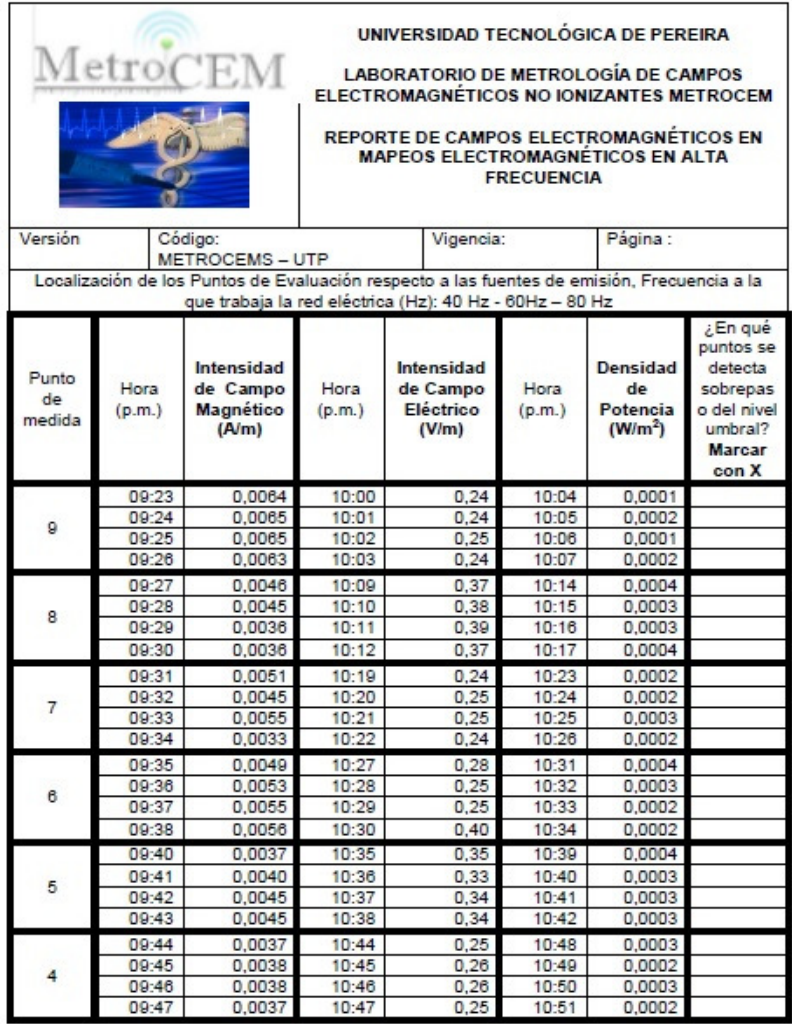

Figura 6. Formato de medida para la consignación de datos.

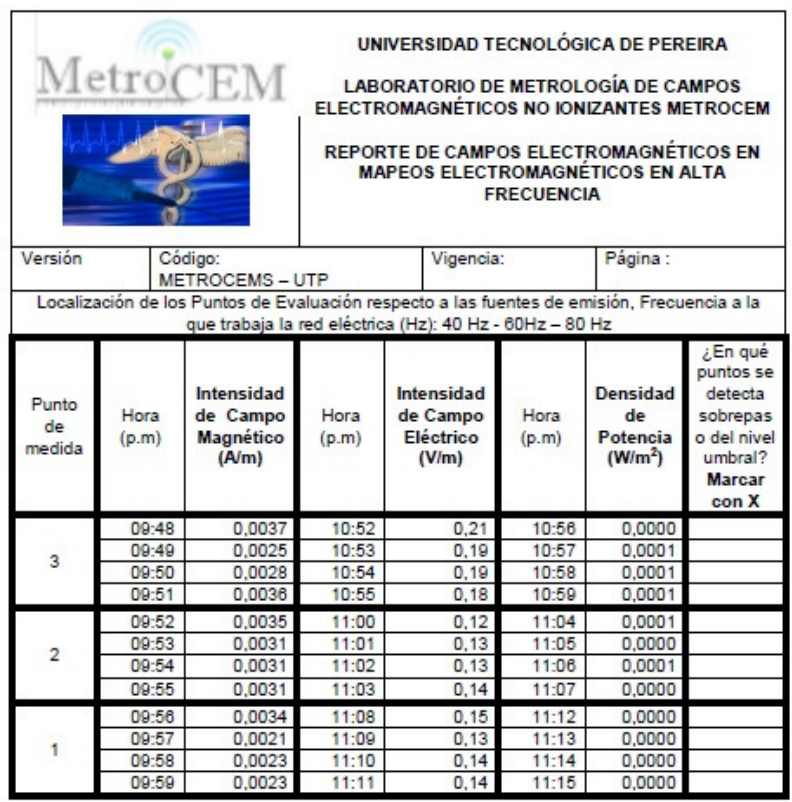

Figura 7. Formato de medida para la consignación de datos.

En la Figura 8 se puede observar el diagrama del mapeo electromagnético con los puntos elegidos en donde se llevó a cabo la medición. En la Figura 9 se representan las valores obtenidos en mapas de contorno, los cuales son comparados directamente con los rangos de exposición permisibles por la 
ICNIRP (observar la tabla 2), la escala de los mapas de contorno muestran las zonas más frías con un tono claro y las más calientes con un tono oscuro.

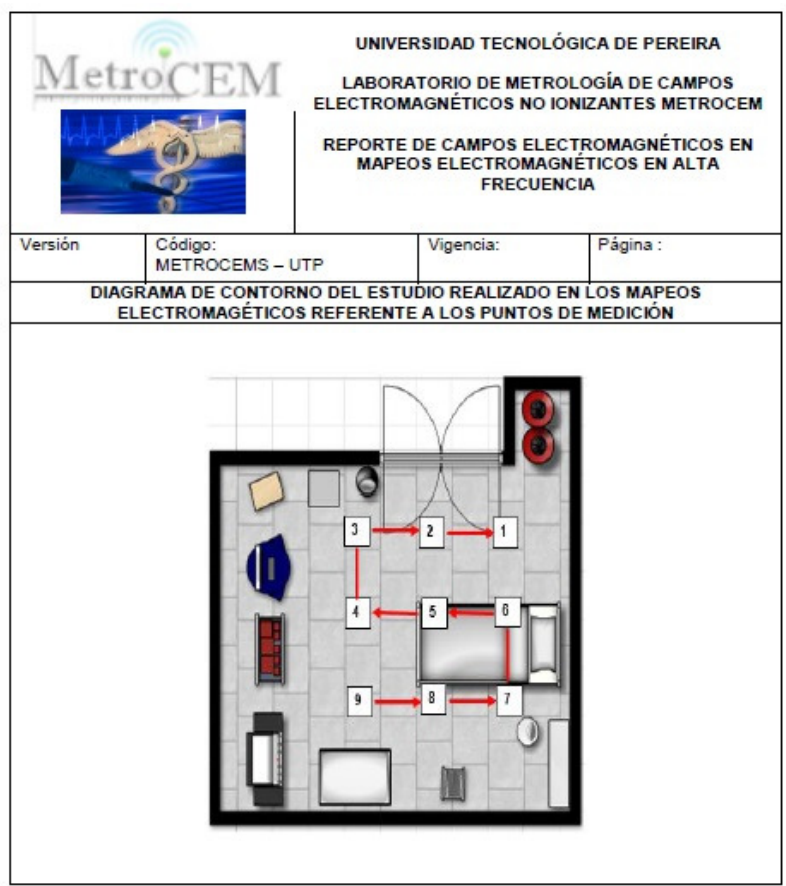

Figura 8. Formato de medida para la consignación de datos.

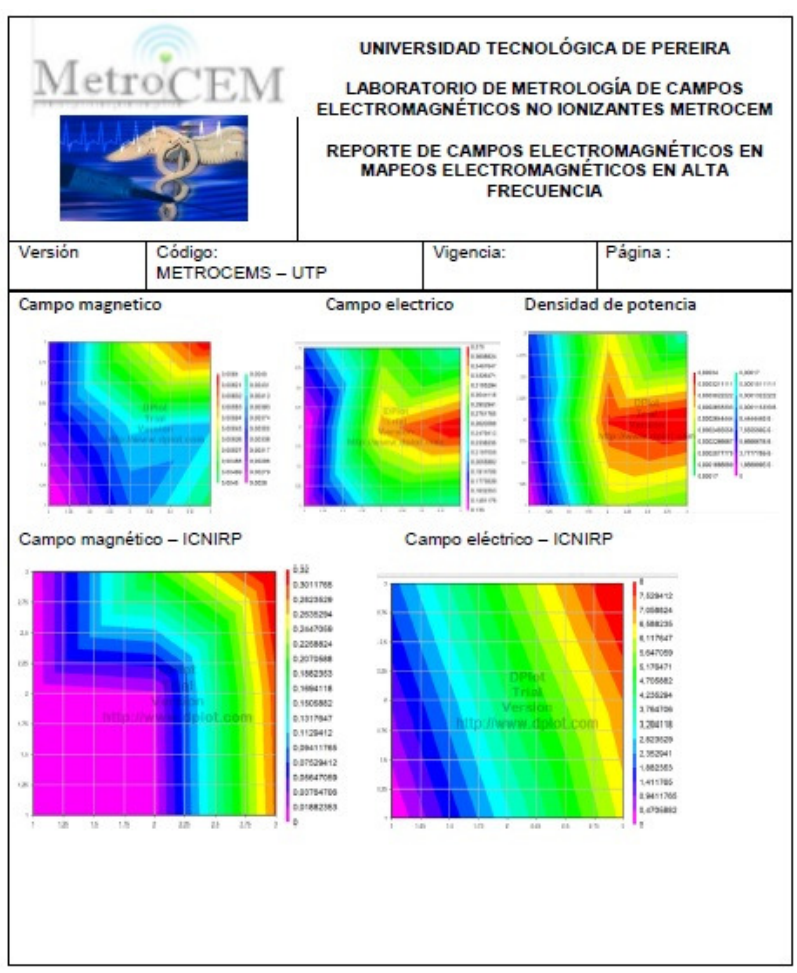

Figura 9. Formato de medida para la consignación de datos.

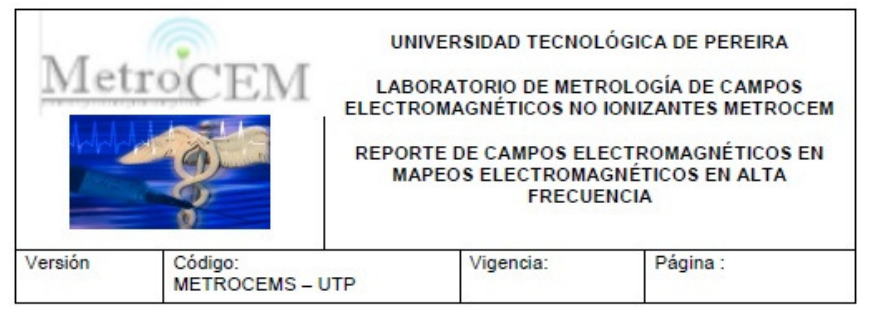

Medición Preliminar

\begin{tabular}{|c|c|c|c|c|c|}
\hline \multicolumn{3}{|l|}{ MAGNITIDES } & \multicolumn{3}{|c|}{\begin{tabular}{|lccc}
$\begin{array}{l}\text { Medida del conjunto } \\
\text { correspondientes tomados. }\end{array}$ & de valores \\
\end{tabular}} \\
\hline \multicolumn{3}{|c|}{ Campo Eléctrico $(\mathrm{E})$} & \multicolumn{3}{|c|}{0.25} \\
\hline \multicolumn{3}{|c|}{ Campo Magnético (B) } & \multicolumn{3}{|c|}{0.0041} \\
\hline \multicolumn{3}{|c|}{ Densidad de Potencia (S) } & \multicolumn{3}{|c|}{0.0002} \\
\hline $\begin{array}{l}\text { Incertidumb } \\
\text { re tipo A } \\
\left(U_{A}\right)\end{array}$ & \begin{tabular}{|l|} 
Incertidumb \\
re tipo B por \\
exactitud \\
$\left(U_{B 1}\right)$ \\
\end{tabular} & \begin{tabular}{|l|} 
Incertidumb \\
re tipo B por \\
Resolución \\
(U⿰冫)
\end{tabular} & \begin{tabular}{|l|} 
Incertidumb \\
re \\
combinada \\
$\left(U_{c}\right)$ \\
\end{tabular} & $\begin{array}{l}\text { Factor } k \text { de } \\
\text { cobertura }\end{array}$ & \begin{tabular}{|l|} 
Incertidumbre \\
expandida $\left(\mathrm{U}_{g}\right)$
\end{tabular} \\
\hline 0.0075 & 0.0058 & 0.0028 & 0.0108 & 1.96 & 0.0212 \\
\hline 0.0002 & 0.0058 & 0.000028 & 0.0058 & 1.96 & 0.0113 \\
\hline 0.000026 & 0.0058 & 0.000028 & 0.0058 & 1.96 & 0.0113 \\
\hline
\end{tabular}

\begin{tabular}{|c|c|c|c|}
\hline $\begin{array}{l}\text { CAMPO } \\
\text { ELÉCTRICO }\end{array}$ & $\begin{array}{l}\text { CAMPO } \\
\text { MAGNÉTICO }\end{array}$ & $\begin{array}{l}\text { DENSIDAD } \\
\text { POTENCIA }\end{array}$ & \\
\hline $\begin{array}{r}E(\mathrm{~V} / \mathrm{m}) \\
0.25 \pm 0.0212\end{array}$ & $\begin{array}{c}(\mathrm{A} / \mathrm{m}) \\
0.0041 \pm 0.0113\end{array}$ & $\begin{array}{c}\left(\mathrm{W} / \mathrm{m}^{2}\right) \\
0.0002 \pm 0.0113\end{array}$ & $\begin{array}{l}\text { 25\% de acuerdo a una } \\
\text { distribución normal }\end{array}$ \\
\hline
\end{tabular}

Figura 10. Formato de medida para la consignación de datos.
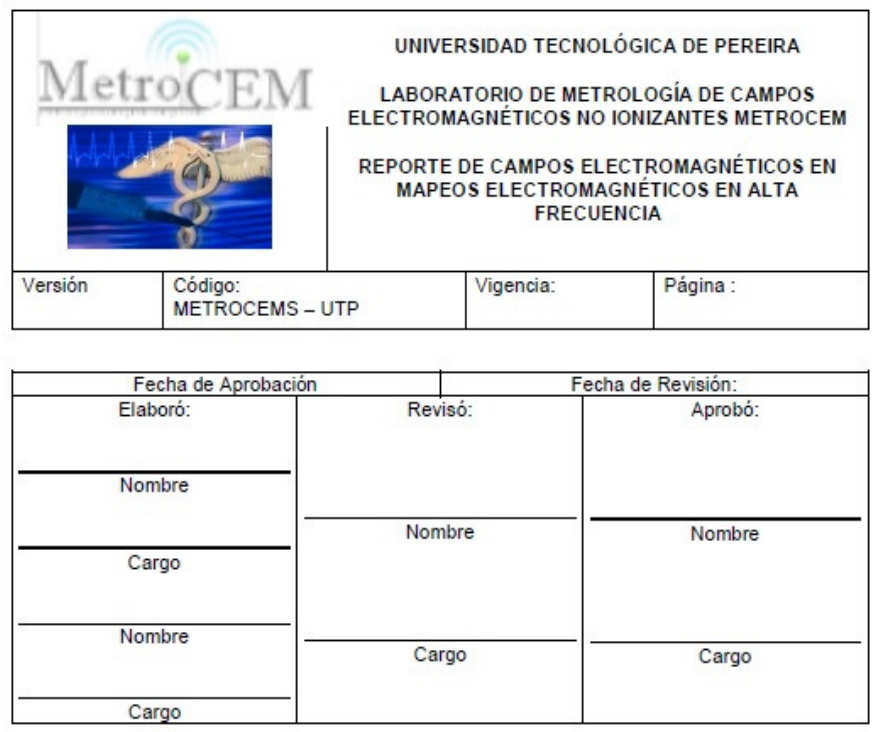

Figura 11. Formato de medida para la consignación de datos. 


\begin{tabular}{|c|c|c|c|c|}
\hline \multicolumn{5}{|c|}{$\begin{array}{l}\text { Niveles de referencia para campos eléctricos, magnéticos y } \\
\text { electromagnéticos }(0 \mathrm{~Hz}-300 \mathrm{GHz} \text {, valores rms imperturbados) }\end{array}$} \\
\hline $\begin{array}{l}\text { Gama de } \\
\text { Frecuencia }\end{array}$ & $\begin{array}{c}\text { Intensidad } \\
\text { de campo } E \\
(\mathrm{~V} / \mathrm{m})\end{array}$ & $\begin{array}{c}\text { Intensidad } \\
\text { de campo H } \\
(\mathrm{A} / \mathrm{m})\end{array}$ & $\begin{array}{c}\text { Campo B } \\
(\mu T)\end{array}$ & \begin{tabular}{|} 
Densidad de \\
potencia \\
equivalente \\
de onda \\
plana \\
$\left(\mathrm{W} / \mathrm{m}^{2}\right)$
\end{tabular} \\
\hline $0-1 \mathrm{~Hz}$ & $\ldots$ & $3,2 \times 10^{4}$ & $4 \times 10^{4}$ & $\ldots$ \\
\hline $1-8 \mathrm{~Hz}$ & 10000 & $3,2 \times 10^{4} / f^{2}$ & $4 \times 10^{4} / f^{2}$ & - \\
\hline $8-25 \mathrm{~Hz}$ & 10000 & $4000 / f$ & $5000 / f$ & - \\
\hline $0,025-0,8 \mathrm{kHz}$ & $250 / f$ & $4 / f$ & $5 / f$ & - \\
\hline $0,8-3 \mathrm{kHz}$ & $250 / f$ & 5 & 6,25 & $\ldots$ \\
\hline $3-150 \mathrm{kHz}$ & 87 & 5 & 6,25 & - \\
\hline $0,15-1 \mathrm{MHz}$ & 87 & $0,73 / f$ & $0,92 / f$ & - \\
\hline $1-10 \mathrm{MHz}$ & $87 / f^{1 / 2}$ & $0,73 / f$ & $0,92 / f$ & - \\
\hline $10-400 \mathrm{MHz}$ & 28 & 0,073 & 0,092 & 2 \\
\hline $400-2000 \mathrm{MHz}$ & $1,375 f^{1 / 2}$ & $0,0037 f^{1 / 2}$ & $0,0046 f^{1 / 2}$ & $f / 200$ \\
\hline $2-3000 \mathrm{GHz}$ & 61 & 0,16 & 0,2 & 10 \\
\hline
\end{tabular}

Tabla 2. Límites de exposición recomendados por la ICNIRP.

Estos protocolos diseñados fueron implementados de igual manera para las mediciones de campos electromagnéticos en baja frecuencia.

Una de las medidas más significativas que se obtuvo fue la realizada en la sala de trabajo de parto, la cual contaba con fuentes de radiación como lo son la incubadora, un equipo de luz para bebes gigantes, un electrocardiógrafo y un monitor de signos vitales, los incrementos en los niveles de intensidad se presentaron debido a que estos equipos médicos se encontraban conectados a la red eléctrica y en funcionamiento, este incremento se dio tanto en la medida para alta frecuencia como para baja frecuencia; es importante aclarar que este aumento no sobrepasó los límites establecidos por los estándares de regulación, sin embargo los resultados entregados a la entidad solicitante deben ser comparados con el documento acompañante de cada equipo electromédico.

\section{CONCLUSIONES}

Se tomaron medidas de campo electromagnético en cuatro salas, donde ninguna de ellas presentó un aumento significativo, encontrándose por debajo del nivel de inmunidad radiada, una de las razones por las cuales no se registran valores representativos se debe a que el hospital cuenta con medidas preventivas en cuanto al uso de sistemas inalámbricos y el uso de teléfonos móviles dentro de este lugar.

De acuerdo con la normatividad existente para la medición de campos electromagnéticos no ionizantes, puede establecerse que en Colombia es necesaria la creación de un organismo acreditado para verificar y certificar los niveles de exposición a CEM-NI, con el fin de que estos niveles no sobrepasen los estándares adoptados por las diversas instituciones internacionales, debido a que en Colombia no existe un laboratorio acreditado que preste este servicio.

La técnica de mapeo electromagnético para la medición de CEM-NI, sirve a las entidades clínico-hospitalarias como información para que estas verifiquen con base en los resultados, que los equipos electromédicos están trabajando en un ambiente electromagnético propicio para su correcto funcionamiento.

Como trabajos futuros se propone que esta metodología sea desarrollada en mayores muestras, recomendando que la mayoría de los equipos electromédicos se encuentren conectados a la red eléctrica y en funcionamiento.

\section{AGRADECIMIENTOS}

A COLCIENCIAS, Departamento Administrativo de Ciencia Tecnología e Innovación por su programa de jóvenes Investigadores e Innovadores 2013-2014 y a la Universidad Tecnológica de Pereira por apoyar dicho programa.

A la Vice-rectoría de investigaciones de la Universidad Tecnológica de Pereira por su apoyo económico al proyecto titulado "CAMPOS ELECTROMAGNÉTICOS NO IONIZANTES - MEDICIÓN, CERTIFICACIÓN, EVALUACIÓN DEL RIESGO Y ESTUDIO PILOTO”.

\section{Conflictos de interés}

Los autores declaran no tener conflictos de interés. 


\section{REFERENCIAS}

[1] Ramos Gonzales Victoria., (2004). Seguridad eléctrica en telemedicina.

En: http://gesdoc.isciii.es/gesdoccontroller?action=download\&id= 24/10/2012-90abf09e23. (Octubre del 2013).

[2] Escobar Adolfo., Moncada María Elena., Guarnizo Cristian., Vergara Viviana., Blandón Jhon., Ariza Gladys., Sierra Liliana., Escudero Natalia. Caracterización de los niveles de campo electromagnético de alta y baja frecuencia en las unidades hospitalarias de la ese metrosalud. En: http://www.metrosalud.gov.co/intra-joomla/images/5.pdf. (Junio del 2013).

[3] Problemática del análisis de interferencias y la compatibilidad electromagnética. En: http://www.coit.es/foro/pub/ficheros/libroscapitulo_3_89e5fa 8e.pdf. (Septiembre del 2013).

[4] Norma Técnica NTC-ISO/IEC Colombiana 17025., (2005). Requisitos generales para la competencia de los laboratorios de ensayo y calibración. En: http://www.itp.gob.pe/normatividad/demos/doc/Normas\%20I nternacionales/Union\%20Europea/ISO/ISO17025Laboratorio Ensayo.pdf. (Junio del 2013).

[5] Norma Técnica Colombiana (GTC 51)., (1997). Guía para la expresión de incertidumbres en las mediciones. En: http: //tienda.icontec.org/brief/GTC51.pdf. (Junio del 2013).

[6] Norma Técnica NTC-ISO/IEC 60601-1-2 Colombiana., (2004). Equipos electromédicos, parte 1: Requisitos generales de seguridad, parte 2: Norma colateral. Compatibilidad electromagnética requisitos y ensayos.

[7] ICNIRP “Comisión Internacional para la Protección contra la Radiación No Ionizante”. Recomendaciones para limitar la exposición a campos eléctricos, magnéticos y electromagnéticos (hasta $300 \mathrm{GHz}$ ). En: http://www.icnirp.de/documents/emfgdlesp.pdf. (Junio del 2013).

[8] NARDA Safety test Solutions, (2011). ELT 400 "Exposure Level Tester" Operating Manual. Alemania. 52 p.

[9] NARDA Safety test Solutions, (2007). NBM 520 "Narda Broadband Field Meter” Operating Manual. Alemania. 56 p.
[10] Llamosa Rincón L.E., Nieto Betancourt N., Villada Castillo J.F., (2011). Fundamentos para el sistema de gestión de un laboratorio de metrología de campos electromagnéticos no ionizantes. Pereira. Universidad tecnológica de Pereira, $168 \mathrm{p}$.

[11] UIT-TK 52 "Unión Internacional de Telecomunicaciones". (2000). Orientación sobre el cumplimiento de los límites de exposición de las personas a los campos electromagnéticos. En: http://www.vivedigital.gov.co/monitoreo-camposelectromagneticos/docs/normaUIT_K52.pdf.

[12] GUM "guide to the expression of uncertainty in measurement"., (2008). Evaluation of measurement data guide to the expression of uncertainty in measurement. En: http://www.bipm.org/utils/common/documents/jcgm/JCGM_1 00_2008_E.pdf. (Julio del 2013).

[13] Llamosa Rincón L.E., Gómez Espíndola J., (2009). Utilización del teorema del límite central en el cálculo de la incertidumbre de medición. En: Scientia et Technica, Vol.43, pp. 289-290.

[14] Llamosa Rincón L.E., Holguín Tabares C.A., Cruz Muñoz B., (2013). Aseguramiento de la calidad de las mediciones en el laboratorio de física. Pereira. Universidad libre seccional Cali, 225p. 